165-173 (2012)

7. Leroux-Berger, M. et al. J. Bone Miner. Res. 26 1543-1553 (2011).

8. St Hilaire, C. et al. N. Engl. J. Med. 364, 432-442 (2011).
9. Lindsay, M. E. \& Dietz, H. C. Nature 473, 308-316 (2011).

10.McDonald, A. I. Cell Stem Cell 23, 210-225 (2018)

This article was published online on 26 September 2018.

\title{
LED technology breaks performance barrier
}

Light-emitting diodes made from perovskite semiconductors have reached a milestone in the efficiency with which they emit light - potentially ushering in a new platform for lighting and display technology. SEE LETTERS P.245 \& P.249

\section{PAUL MEREDITH \& ARDALAN ARMIN}

$\mathrm{L}$ ight-emitting diodes (LEDs) have revolutionized lighting and displays, not least because they use energy more efficiently than any previous light-emitting technology. Micro-LEDs made from inorganic, 'compound' semiconductors are emerging that deliver unprecedented resolution for displays, whereas organic semiconductor LEDs (OLEDs) provide unparalleled colour quality and near- $180^{\circ}$ viewing angles, and could potentially be used to develop flexible, lightweight displays. In this issue of Nature, two papers ${ }^{1,2}$ report what could be the birth of a new family of LEDs based on semiconductors called perovskites. Remarkably, the efficiencies with which the perovskite LEDs (PLEDs) produce light from electrons already rival those of the best-performing OLEDs $^{3}$, and have been achieved in less than four years since the report ${ }^{4}$ of the first PLED suggesting that there is plenty of room for even further improvement in their performance.

Perovskites have shot to scientific stardom in the past few years, mostly because they show great promise for solar cells ${ }^{5}$, but their potential for use in other applications, such as light sensors ${ }^{6}$ and LEDs ${ }^{4}$, is rapidly emerging. Crucially, perovskites can be processed from solution (for example, using low-cost, low-tech printing methods), and work well in the designs for optoelectronic devices that are easiest to make. This might allow perovskitebased devices that have large areas (several square centimetres) to be made extremely cheaply, and with low embodied energy (the total energy involved in the entire life cycle of a device).

Cao et al. ${ }^{1}$ (page 249) and Lin et al. ${ }^{2}$ (page 245) have independently developed PLEDs that break an important technological barrier: the external quantum efficiency (EQE) of the devices, which quantifies the number of photons produced per electron consumed, is greater than $20 \%$. There are several similarities between the devices reported by the two groups. Perhaps most notably, the active (emissive) perovskite layer is about 200 nanometres thick in both cases, and is sandwiched between two relatively simple electrodes. This design is called a planar structure, and is the most basic manifestation of diodes made from thin films of materials (Fig. 1). The electrodes are appropriately modified to ensure that electrons and holes (quasiparticles formed by the absence of electrons in atomic lattices) are efficiently pumped into the perovskite. As in all LEDs, when electrons meet holes, they can release energy in the form of photons through a process known as radiative recombination.

Another similarity between the devices is that the perovskite layers were prepared using solutions, from which the semiconductors crystallized to form the emissive components of the LEDs. Cao et al. used a perovskite known as formamidinium lead iodide (FAPI), mixed with an amino-acid additive (aminovaleric acid) to control the size and orientation of the resultant perovskite crystals. FAPI has been quite widely explored as a semiconductor for solar cells, but Lin et al. report a new composite material in which crystals of the perovskite $\mathrm{CsPbBr}_{3}$ (Cs, caesium; $\mathrm{Pb}$, lead; $\mathrm{Br}$, bromine) are partly enclosed by a shell of an organic compound (methyl ammonium bromide; $\mathrm{MABr}$ ).

Achieving high EQEs in any LED requires the elimination of non-radiative losses - electron-hole-recombination pathways that do not produce photons. Both Cao and colleagues' and Lin and colleagues' PLEDs deliver on this equally well. But the two groups also used other, subtly different methods to improve the EQE.

Cao et al. targeted the outcoupling problem, which is well known to those working with thin-film LEDs (such as PLEDs and OLEDs). The outcoupling problem is that the optical physics of planar diodes causes $70-80 \%$ of the light generated by the semiconductor to be

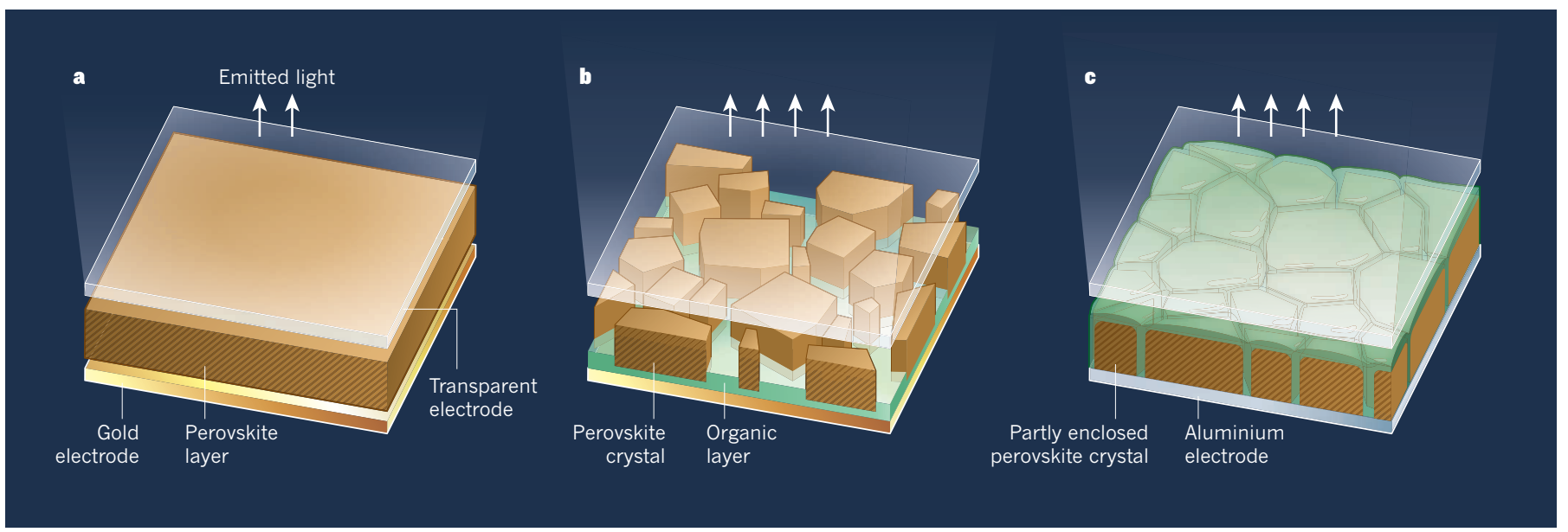

Figure 1 | Improved light-emitting diodes (LEDs) based on perovskite semiconductors. a, LEDs have previously been made from perovskites by sandwiching a thin layer of the semiconductor between a gold electrode and a transparent electrode. However, only about $20 \%$ of the light generated in the perovskite escapes from the device. $\mathbf{b}$, Cao et al. ${ }^{1}$ report perovskite LEDs (PLEDs) in which the semiconductor layer consists of separated submicrometre-sized crystals, partitioned from the gold electrode by a thin layer of an organic material. This design increases the amount of light that escapes. c, Lin et al. ${ }^{2}$ report PLEDs based on a different perovskite, in which the semiconductor crystals are partly enclosed by an organic compound and the gold electrode is replaced by an aluminium one. This device optimizes the efficiency with which charges (not shown) that are pumped into the perovskite are converted into photons. 
trapped in the device. Various strategies have attempted to address this issue in OLEDs, such as using diffraction gratings ${ }^{7}$ and buckling the device ${ }^{8}$.

But Cao and colleagues took a simpler approach: they optimized their perovskiteprocessing conditions so that the emissive layer spontaneously forms as distinct submicrometre-scale crystal platelets (Fig. 1). The authors' computational modelling shows that this submicrometre structuring increases the fraction of light that makes it out of the emissive layer to $30 \%$, compared with $22 \%$ for an equivalent 'flat-layer' perovskite device (a device in which the perovskite layer does not have submicrometre structuring). In combination with the reduction in non-radiative losses, this results in an EQE of 20.7\%.

By contrast, Lin et al. used a flat emissive layer, but tried to optimize the balance of electrons and holes injected into the perovskite, to make the most efficient use of every charge. This seems to be facilitated by the MABr shells that enclose the perovskite crystals. The resulting PLEDs have an EQE of $20.3 \%$.

But caution is advised before ordering your PLED ultrahigh-definition television. OLEDs, and indeed all optoelectronic devices based on organic semiconductors, suffered for many years from stability issues. The first polymer OLEDs ${ }^{9}$ could emit light for only seconds, and subsequent advances were needed to ensure that smartphone screens and OLED televisions last for tens of thousands of hours. The lifetime of LEDs can be measured by the $T_{50}$ metric, which is the time for the performance of the device to drop by half. The $T_{50}$ values of Cao and colleagues' and Lin and colleagues' PLEDs are currently modest: 20 hours and 100 hours, respectively.

Furthermore, displays require a minimum of three colours (and preferably more) to create high-quality colour images. Developing a range of colours for OLEDs was a big challenge. Cao and co-workers' PLED emits in the near-infrared region of the electromagnetic spectrum, and Lin and co-workers' PLED emits green light - which is definitely a good start. Multiple colours of PLEDs could be generated by altering the composition of the devices, but the same developmental journey as was needed for OLEDs lies ahead.

The two papers also highlight problems that occur every time new optoelectronic materials emerge as a technological platform: inconsistent characterization and a lack of standards. Because Cao and colleagues' PLED emits light from outside the visible spectrum, they report the metrics of their devices radiometrically - they use a measure that simply takes into account the total emitted power. By contrast, Lin and colleagues describe the emission of their green PLED using photometric measures, which are weighted by the response of the human eye. The two groups also report the peak EQEs at different brightnesses, and therefore at different driving currents. This makes direct comparison somewhat problematic.

Caveats aside, the two papers are a milestone in PLED development. For now, LEDs based on compound semiconductors remain the dominant technology: they outclass the competition in many respects, including cost, efficiency, colour and brightness. They will be hard to beat. But that should not stop the pioneers of perovskite (or, indeed, organic) LEDs from trying.

Paul Meredith and Ardalan Armin are in the Department of Physics, Swansea University, Singleton Park, Swansea SA2 8PP, UK. e-mails:paul.meredith@swansea.ac.uk; ardalan.armin@swansea.ac.uk

1. Cao, Y. et al. Nature 562, 249-253 (2018).

2. Lin, K. et al. Nature 562, 245-248 (2018)

3. Di, D. et al. Science 356, 159-163 (2017).

4. Tan, Z.-K. et al. Nature Nanotechnol. 9, 687-692 (2014).

5. Lee, M. M., Teuscher, J., Miyasaka, T., Murakami, T. N \& Snaith, H. J. Science 338, 643-647 (2012)

6. Lin, Q., Armin, A., Burn, P. L. \& Meredith, P. Nature Photon. 9, 687-694 (2015).

7. Ziebarth, J. M., Saafir, A. K., Fan, S. \& McGehee, M. D. Adv. Funct. Mater. 14, 451-456 (2004).

8. Koo, W. H. et al. Nature Photon. 4, 222-226 (2010).

9. Burroughes, J. H. et al. Nature 347, 539-541 (1990).

\section{ANIMAL BEHAVIOUR}

\section{Foraging skills develop over generations}

The movements of relocated wild animals reveal that a lost migratory skill was regained over successive generations. This suggests that skill improvements can occur over time as animals learn expertise from each other.

\section{ANDREW WHITEN}

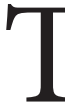
The transmission of behavioural traditions by learning from others - cultural learning - was once thought to be a uniquely human attribute. However, evidence increasingly indicates that this phenomenon is widespread among animals, shaping behaviours from foraging for food to mate choice to predator avoidance ${ }^{1}$. Claims for human uniqueness in our cultural skills have therefore been pinned on our species' capacity for what is called cumulative culture: the ramping up of cultural sophistication as each generation builds on their ancestors' cultural achievements ${ }^{2,3}$. Writing in Science, Jesmer et al. ${ }^{4}$ now challenge this view in a study of the development of migratory skill in wild populations of bighorn sheep (Ovis canadensis) and moose (Alces alces) populations that have been moved to unfamiliar locations. Their findings have implications for understanding the evolution of cumulative culture in both humans and other animals, and for conservation policies ${ }^{5,6}$.

In the wild, bighorn sheep (Fig. 1) and moose normally migrate in spring and move between distinct seasonal ranges. These movements follow a pattern known as green-wave surfing, whereby the animals' migration tracks the availability of high-quality vegetation, which peaks at different times in different places depending on factors such as altitude. How animals evolved the capacity for this type of migratory behaviour remains unknown.

Jesmer and colleagues investigated the migration of bighorn sheep and moose that had been moved to unfamiliar areas in recent decades to repopulate regions in which these types of animal had been wiped out by disease or hunting. The authors compared the migration of such relocated populations with that of animals in long-established populations that had been migrating for many generations in a particular region. They noted that when individuals had been moved to an unfamiliar location, the animals usually ceased migrating, although migratory behaviour gradually re-emerged in subsequent generations.

The researchers fitted animals with a collar containing a Global Positioning System (GPS) device that enables accurate tracking of an animal's position. They combined this information with the corresponding satellite imagery for the region that revealed where and when vegetation was at peak quality. To measure animals' green-wave surfing skills, the authors counted the number of days between the peak forage quality at a location and the arrival of an animal there. When the authors analysed bighorn sheep from migratory populations that had been relocated at times ranging from 0 to 35 years ago, these animals surfed the green wave approximately half as effectively as animals from populations that had been established in a particular region for more than 200 years.

Jesmer and colleagues then combined these and other bighorn records with similar data for moose that had been relocated to a given region between 10 and 110 years ago. The combined results for these $267 \mathrm{big}$ horn and 189 moose were consistent with a model in which it took up to 30 years (between 4 and 5 generations) for migration to distinct 